Service social

\title{
Le Temps d'y voir - Conférence internationale sur la situation des filles, sous la direction de Marguerite Séguin-Desnoyers, Montréal, Guérin, 1986, 351 pages.
}

\section{Marie Drolet}

Volume 35, numéro 3, 1986

Les jeunes et le travail social

URI : https://id.erudit.org/iderudit/706327ar

DOI : https://doi.org/10.7202/706327ar

Aller au sommaire du numéro

Éditeur(s)

École de service social de l'Université Laval

ISSN

1708-1734 (numérique)

Découvrir la revue

Citer ce compte rendu

Drolet, M. (1986). Compte rendu de [Le Temps d'y voir-Conférence internationale sur la situation des filles, sous la direction de Marguerite Séguin-Desnoyers, Montréal, Guérin, 1986, 351 pages.] Service social, 35(3), 480-481. https://doi.org/10.7202/706327ar d'utilisation que vous pouvez consulter en ligne.

https://apropos.erudit.org/fr/usagers/politique-dutilisation/ 
Le Temps d'y voir - Conférence internationale sur la situation des filles, sous la direction de Marguerite SÉGUIN-DESNOYERS, Montréal, Guérin, 1986, 351 pages.

Le Temps d'y voir réunit la majorité des textes présentés à la Conférence internationale sur la situation des filles, tenue à Montréal, les 29, 30 et 31 octobre 1985. Organisée par la Fondation Marie-Vincent et l'Université de Montréal, cette réunion voulait souligner l'Année internationale de la jeunesse et la dernière année de la décennie des femmes.

Dès la préface, Madame Marguerite Séguin-Desnoyers, présidente du comité organisateur, clarifie s'il s'agit d'une conférence féministe: "Si parler des filles d'aujourd'hui, de la société qui doit et devra rencontrer leurs aspirations, des structures qui entravent la réalisation de leurs espérances crée un lien avec le féminisme, alors oui, elle s'inscrirait dans ce mouvement ". Elle signale que la Conférence s'est inspirée de travaux qui ont soutenu la démarche des femmes vers l'égalité et l'autonomie. Le recueil regroupe notamment des allocutions d'écrivaines féministes de renom comme Benoîte Groulx, Françoise Collin et Flora Groulx et présente aussi le point de vue de certaines chercheuses et d'auteures s'identifiant comme féministes.

Madame Séguin-Desnoyers note que les aspirations scolaires, professionnelles et familiales des adolescentes occidentales présentent toujours un caractère traditionnel. Elles optent majoritairement pour les carrières "dites féminines", comme les sciences humaines, les sciences paramédicales, le secrétariat, l'éducation et même le service social. Les adolescentes choisissent moins les professions orientées vers les sciences et les mathématiques qui sont pourtant les « secteurs les plus prometteurs pour l'avenir ». Elles sont peu conscientes de la réalité du marché du travail et espèrent une vie affective stable avec mariage et enfants. "On ne peut certes reprocher aux jeunes de rêver d'un amour idéal mais il ne faut pas que ces rêves masquent la réalité et les empêchent de s'engager dans les voies de la réussite. $"$

Ces préoccupations ont motivé la tenue du colloque, influencé le choix des présentations et se reflètent dans le recueil des actes de la Conférence. En effet, plus de la moitié des trente-quatre textes présentés touchent ce sujet, que ce soit par des études démontrant cet état de fait, par des commentaires sur cette situation ou par des tentatives d'intervention pour contrer cette direction.

Les autres auteurs apportent diverses données sur la problématique des adolescentes de la première moitié des années quatre-vingt. Le recueil diffuse d'intéressantes informations récentes et des réflexions à la fois sur : l'identification féminine, le rôle du père, la sexualité, les maladies transmises sexuellement, la prostitution juvénile, les abus sexuels et la situation des jeunes filles immigrantes.

La plupart des textes offrent une liste fouillée de références bibliographiques sur chaque sujet. II devient ainsi un ouvrage de référence à retenir pour ses données pertinentes; il éveille aussi la discussion sur la situation actuelle des filles, leur socialisation et leurs contraintes particulières dans la société moderne 
déjà difficile pour tout jeune. Mais ce cadre global et le contexte sociétal sont moins analysés.

Nous ne pourrions terminer cette présentation des actes du colloque d'octobre 1985 sans signaler la polémique qui a entouré la Conférence ellemême. En effet, les journaux québécois ont déploré l'absence des adolescentes aux discussions et ils ont mis en évidence le malaise identifié dans les propos des participantes. Même si la plupart se disaient confiantes dans le potentiel et la créativité des jeunes pour défendre leurs droits et créer leur place, «la peur et la culpabilité " ont tout de même transparu. Comme l'a fait remarquer Benoîte Groulx dans le discours d'ouverture, il ne faudrait pas que les adolescentes "se laissent tenter par l'individualisme et s'endorment sur les victoires de leurs mères".

CLSC Les Forges,

Marie Drolet

Trois-Rivières.

L'abus des drogues - Les programmes de prévention chez les jeunes, par Line Beauchesne, Sillery, Presses de l'Université du Québec, 1986, 106 pages. ("Monographie de psychologie».)

Tant une recherche spécifique peut représenter, à un moment donné, une percée dans un domaine particulier, tant une recension des écrits peut apparaître comme un moment de synthèse et de réflexion dans l'évolution scientifique. C'est à ce dernier aspect que se rattache le présent ouvrage. Celui-ci a été produit grâce à une recherche subventionnée du Conseil québécois de la recherche sociale dont l'objet comprenait deux volets : la présentation critique des enquêtes sur l'usage et l'abus des drogues chez les jeunes au Québec dans les années 1980, et une synthèse critique des recherches évaluatives sur les programmes de prévention.

Le premier chapitre a trait aux différentes enquêtes sur l'usage des drogues au Québec. Trois recherches produites dans la région de Montréal sont donc examinées à l'aide des critères scientifiques validés par l'Organisation mondiale de la santé. Les conclusions qui découlent de cet examen invitent à la prudence : données trop limitées pour saisir la problématique, bases méthodologiques qui ne permettent pas la comparaison, problèmes conceptuels quant à la définition de l'abus de consommation.

Les recherches évaluatives sur l'efficacité des programmes de prévention constituent le second chapitre. Elles abordent la modification de l'usage effectif ou des intentions d'usage des drogues, et des attitudes des jeunes à l'égard des drogues. Encore ici, on se heurte à des faiblesses méthodologiques importantes (grandeur des échantillons, validité et fiabilité des instruments de mesure) et la seule indication retenue a trait à la plus grande efficacité des programmes de prévention à modèles multiples (combinaison de plusieurs interventions à des niveaux différents) par rapport aux programmes à modèle unique. 